\title{
Clinical Predictors of Arterial Extravasation, Rebleeding and Mortality Following Angiographic Interventions in Gastrointestinal Bleeding
}

\author{
Prasoon Mohan ${ }^{1}$, John Manov ${ }^{2}$, Alexander Diaz-Bode ${ }^{3}$, Sree Venkat ${ }^{1}$, Michael Langston ${ }^{4}$, Akash Naidu ${ }^{2}$, Rayna Howse ${ }^{1}$, \\ Govindarajan Narayanan ${ }^{1}$
}

1) Department of

Interventional Radiology,

University of Miami,

2) Department of Radiology,

University of Miami,

3) University of Miami School

of Medicine,

4) Department of Medicine,

University of Florida,

Miami, FL, USA

\begin{abstract}
Background \& Aims: The aim of this study was to identify clinical and imaging predictors of arterial extravasation, post embolization rebleeding and 30-day mortality in gastrointestinal (GI) bleeding. Method: This retrospective study included 114 patients who underwent angiography for upper or lower GI bleeding. Multivariate logistic regression was used to identify clinical and imaging predictors.

Results: Angiography demonstrated arterial extravasation in 22 patients (19\%) and embolization was performed in 48 (42\%) patients including prophylactic embolization in $26(56 \%)$. Fall in hemoglobin level from baseline was an independent predictor of arterial extravasation with $65 \%$ increased odds for every unit drop (OR 1.65, 95\%CI 1.13-2.40, $\mathrm{p}=0.01$ ). Age $<60$ years was a negative predictor of rebleed within 30 -days (OR $0.94,95 \% \mathrm{CI} 0.89-1.00, \mathrm{p}=0.04$ ). Patients with a history of malignancy were more likely to rebleed (OR $4.4,95 \%$ CI 1.06-18.36, $\mathrm{p}=0.04$ ). Hemodynamic instability prior to angiography (OR 13.22, 95\%CI 1.65-106.07, $\mathrm{p}=0.02$ ), history of malignancy (OR $1.36,95 \% \mathrm{CI} 1.49-10.49, \mathrm{p}=0.01)$, number of units of platelets transfused (OR 1.42, 95\%CI 1.02-1.97, $\mathrm{p}=0.04$ ) and rebleed after angiography (OR 46.8, 95\%CI 4.80-456.14, $\mathrm{p}<0.01$ ) were predictors of 30-day mortality. Prophylactic embolization was not a predictor of rebleed or 30-day mortality. Conclusions: This paper identified important clinical predictors of arterial extravasation, rebleed and 30-day mortality in GI bleedings, which will assist in patient selection and help to improve the overall angiographic management of GI bleeding.
\end{abstract}

Key words: Embolization - upper gastrointestinal bleeding - lower gastrointestinal bleeding - mesenteric angiography.

Abbreviations: CT: computed tomography; CTA: computed tomography angiography; FFP: fresh frozen plasma; GI: gastrointestinal; INR: International Normalized Ratio; IRB: institutional review board; PACS: Picture Archiving and Communication System; pRBC: packed red blood cells; PT: prothrombin time; TAE: transcatheter angiographic embolization.

\section{INTRODUCTION}

Acute gastrointestinal (GI) bleeding results in over 300,000 hospitalizations annually in the United States, which amounts to $1-2 \%$ of all admissions [1]. The costs of inpatient management of acute GI bleeding approach $\$ 1$ billion dollars each year [2]. Upper GI bleeding is responsible for most of these admissions, estimated at 100 cases per 100,000 persons [3]. Lower GI tract bleeding has a much lower incidence of 20-27 per 100,000, representing only $24 \%$ of all GI bleedings [3]. Persons over the age of 60 represent $35-45 \%$ of all cases, and this proportion continues to increase [3].

The significance of GI bleeding is underscored by a mortality rate of 5-12\%, which has not changed significantly in the past five decades [4]. Endoscopic intervention remains the primary modality for treatment of non-variceal GI bleeding. When endoscopic intervention fails or is not possible, angiographic or surgical intervention is warranted $[3,5]$. Angiographic intervention is preferred over surgical treatment as it preserves the bowel and is minimally invasive. A major limitation for transcatheter angiography is the high incidence of negative studies that fail to identify or localize the bleeding [6]. Previous studies have shown negative angiograms in as many as $52 \%$ of cases [7]. This makes appropriate selection of patients for angiographic intervention a critical component 
in the management of GI bleeding. Another limitation of angiographic intervention is the incidence of rebleeding after embolization, estimated to be about 33\% for upper GI and $21 \%$ for lower GI bleeding $[8,9]$. Identification of patients at risk of rebleeding would greatly help in the better management of these patients. The aim of this study was to identify any clinical or imaging factors that predict a positive angiogram in non-variceal GI bleeding. Secondary aims of the study were to identify any clinical or imaging predictors of rebleeding after embolization and 30-day mortality.

\section{MATERIAL AND METHODS}

This is an IRB approved retrospective study of 114 patients from two institutions, who underwent angiography for GI bleeding. Patients were identified using billing and coding data from $12 / 2010$ to $12 / 2015$. Inclusion criteria included all cases of upper or lower, non-variceal bleeding across all age groups and genders, admitted for angiography. All patients underwent endoscopy prior to angiography. Exclusion criteria were cases of variceal bleeding or patients with cirrhosis. Data were extracted from electronic medical records, PACS and outside imaging. All data were collected and analyzed by five of the authors.

Collected data included patient demographics, hemodynamic parameters, baseline hemoglobin, coagulation studies, platelets, blood type, transfusions, CT angiogram and nuclear medicine study findings, lowest hemoglobin $\mathrm{Hb}$ ) recorded within the 24 hours preceding the procedure, anticoagulation if any, intervention type, angiographic findings including interventions, embolization material, occurrence of rebleeding and 30-day mortality.

A multivariate regression analysis was used for the identification of any factors that predicted positive angiogram, rebleed or 30-day mortality. Linear regression analysis allowed for identification of several independent variables and their relationship to our dependent variables, in this case positive angiogram, rebleed, or 30-day mortality. IBM SPSS software was used for all statistical analysis. Multiple independent variables comprising patient age, site of GI bleed, history of malignancy, anticoagulation therapy, $\mathrm{Hb}$ on admission, drop in $\mathrm{Hb}$ from baseline, platelet count, recent transfusion, hemodynamic instability, and rebleed status were input into the software to compare to dependent variables which included positive angiogram, rebleed, and 30-day mortality. Chi squared test and $t$-test were used for analysis of categorical and continuous variables respectively.

\section{RESULTS}

\section{Patient background}

Data collection resulted in an unselected, consecutive cohort of 114 patients who underwent angiography between $12 / 2010$ to $12 / 2015$ for upper or lower GI bleeding. The mean age of patients was 63 years (SD 17.1) and 59 (52\%) were female. Clinical examination localized the site of bleeding to the upper GI tract in 57 (50\%) patients, the lower GI tract in 54 (47\%) patients, and in $3(3 \%)$ patients the site was not localized. Thirty-six (32\%) had a documented history of malignancy, and $13(11 \%)$ presented malignancies of gastrointestinal organs. Eight (7\%) patients were on warfarin therapy and $3(3 \%)$ were on heparin at the time of angiography (Table I). Final diagnosis was upper GI bleeding in 10 (9\%), lower GI bleeding in $20(18 \%)$, malignancy in $15(13 \%)$, ulcers in $14(12 \%)$, Dieulafoy lesion in $2(2 \%)$, duodenal hematoma in $2(2 \%)$, pseudoaneurysm in $5(4 \%)$, diverticulitis in 15 (13\%), angiodysplasia in $3(3 \%)$, segmental arterial mediolysis syndrome in $1(1 \%)$, unspecified in $24(21 \%)$, related to a dive injury in $1(1 \%)$, and esophageal perforation in $1(1 \%)$.

Table I. Characteristics of patients undergoing angiography for clinically suspected GI bleeding

\begin{tabular}{lcc}
\hline & Mean & SD or \% \\
\hline Age (years) & 63 & $17.1^{\star}$ \\
Female & 59 & $52 \%$ \\
History of malignancy & 36 & $32 \%$ \\
Nadir $\mathrm{Hb}(\mathrm{g} / \mathrm{dL})$ & 7.2 & $1.5^{\star}$ \\
Drop in $\mathrm{Hb}(\mathrm{g} / \mathrm{dL})$ & 3.6 & $2.2^{\star}$ \\
pRBCs transfused (units) & 4.96 & $5.6^{\star}$ \\
\hline
\end{tabular}

*: Standard Deviation; pRBCs: packed Red Blood Cells; Hb: Hemoglobin.

\section{Patient status at angiography}

Mean nadir of $\mathrm{Hb}$ during admission was $7.2 \mathrm{~g} / \mathrm{dL}$ (SD 1.5 ) with a mean baseline $\mathrm{Hb}$ of $10.7 \mathrm{~g} / \mathrm{dL}$ (SD 1.7); the mean drop in $\mathrm{Hb}$ was $3.6 \mathrm{~g} / \mathrm{dL}$ (SD 2.2). Mean platelet count was $123,000 / \mu \mathrm{L}$ (SD 86,000). Mean INR was 1.28 (SD 0.47). In the 24 hours prior to angiography, 55 (60\% of 92 with data available) required transfusion of packed red blood cells (pRBCs) and they received a mean 4.96 units (SD 5.6). Twentyfive of $86(29 \%)$ patients required platelet transfusion prior to angiography with a mean of 2.4 units (SD 1.9) transfused. Fresh Frozen Plasma (FFP) was required in 45 of 86 (52\%) and mean of 12.2 units (SD 23) was transfused. Patients were considered hemodynamically unstable if they were on vasopressors or had a systolic BP less than $90 \mathrm{mmHg}$. At the time of angiography, $35(41 \%)$ patients were hemodynamically stable, and afterward $47(57 \%)$. There was a significant decrease in the proportion of hemodynamically unstable patients after angiography $(\mathrm{p}<0.01)$.

\section{Angiography}

Angiography demonstrated extravasation in 22 patients (19\%), 12 of these were upper GI bleedings and 10 were lower GI bleedings. Embolization was performed on a total of 48 (42\%) patients. Technical success was achieved in $98 \%$ of cases, with only 1 out of 48 patients demonstrating blood flow distal to the coil. Coils were the most commonly used material for embolization, which were used in $41(87 \%)$ patients. Coils alone were used in 27 (57\%), coils with gelfoam in $14(30 \%)$, gelfoam alone in $6(13 \%)$ and vascular plug in one case. Prophylactic embolization was done in 26 patients (56\%). The vessel for prophylactic embolization was selected based on endoscopy, CT angiogram or nuclear medicine (NM) findings. On logistic regression, the fall in $\mathrm{Hb}$ from baseline was predictive of arterial extravasation on angiogram (OR 1.65, 95\% CI 1.13$2.40, \mathrm{p}=0.01)$. Other hematological parameters such as platelet count, PT, or INR were not predictive of extravasation $(\mathrm{p}=0.98$, 
$0.15,0.05$, respectively). The quantities of pRBCs, platelets, or FFP transfused were not predictive of positive angiogram ( $\mathrm{p}=0.99,0.99,0.64$, respectively) (Table II).

Table II. Results of angiography performed on patients with clinically suspected GI bleeding

\begin{tabular}{lcc}
\hline & Number of Patients & $\%$ \\
\hline Active arterial extravasation & 22 & $15 \%$ \\
Prophylactic embolization & 26 & $58 \%$ \\
Embolization material & & \\
$\quad$ Coils & 27 & $57 \%$ \\
Coils+Gelfoam & 14 & $30 \%$ \\
Gelfoam & 6 & $13 \%$ \\
$\quad$ Vascular plug & 1 & $2 \%$ \\
\hline
\end{tabular}

Seventeen patients (15\%) underwent CTA prior to angiography with 5 (29\%) positive studies. Forty-two patients (37\%) underwent 99-Tc-tagged red blood cell scan prior to angiography and 26 (62\%) were positive. Using logistic regression, neither positive CTA nor scintigraphy were predictive of demonstration of extravasation on angiography (CTA: OR 2.0, 95\% CI 0.21-18.33, p=0.54; scintigraphy: OR $0.79,95 \%$ CI $0.15-4.09, \mathrm{p}=0.78)$.

\section{Post angiography course}

After angiography, the mean length of stay was 20.7 days (SD 40). Rebleeding occurred within 30-days in 14 of the 41 patients for whom follow-up data were available. There were $22(20 \%)$ deaths within 30 days. Younger patients $(<60$ years) were significantly less likely to have rebleeding within 30-days (OR 0.94, 95\%CI 0.89-1.00, p=0.04). Patients with a history of malignancy were more likely to rebleed within 30-days (OR 4.4, 95\%CI 1.06-18.36, p=0.04). There was not a significant difference in the rate of rebleeding between upper or lower GI source of bleeding. (OR 1.11, 95\%CI 0.29-4.31, $\mathrm{p}=0.88$ ). Material used during embolization did not predict the likelihood of recurrent GI bleed within 30-day follow-up $(\mathrm{p}=0.81)$ (Table III).

Table III. Potential predictors of rebleeding occurrence within 30 days of endovascular embolization in patients with clinically determined GI bleeding

\begin{tabular}{lcc}
\hline Parameter & OR & $95 \%$ CI \\
\hline Age & 0.94 & $0.89-1.00$ \\
Location & 1.11 & $0.29-4.31$ \\
Prophylactic embolization & 1.44 & $0.39-5.27$ \\
Material & NA & $\mathrm{p}=0.81^{\star}$ \\
\hline
\end{tabular}

${ }^{*} \mathrm{p}$-values given where $95 \%$ CI not applicable

Rebleeding within 30 days of angiography was predictive of 30-day mortality (OR 46.8, 95\%CI 4.80-456.14, p<0.01.). Hemodynamic instability at the time of angiography was found to be a predictor of 30-day mortality (OR 13.22, 95\%CI 1.65-106.07, $\mathrm{p}=0.02$ ). Presence of a known malignancy was associated with increased 30-day mortality (OR 1.36, 95\%CI $1.49-10.49, \mathrm{p}=0.01)$. Interestingly, the number of units of platelets transfused was a predictor of 30-day mortality (OR 1.42, 95\%CI 1.02-1.97, p=0.04). Prophylactic embolization was not associated with increased mortality at 30-days (OR $1.47,95 \%$ CI $0.37-5.96, \mathrm{p}=0.59$ ), nor was it associated with an increased risk of rebleeding within 30 days (OR 1.44, 95\%CI 0.39-5.27, $\mathrm{p}=0.59$ ). There was not a significant difference in mortality between upper, lower, and clinically unlocalized gastrointestinal bleeding $(\mathrm{p}=0.19)$ (Table IV).

Table IV. Potential predictors of 30-day mortality in patients undergoing endovascular embolization for clinical GI bleeding.

\begin{tabular}{lcc}
\hline Parameter & OR & $95 \% \mathrm{CI}$ \\
\hline Rebleed & 46.8 & $4.80-456.14$ \\
History of malignancy & 1.36 & $1.49-10.49$ \\
Location & $\mathrm{NA}$ & $\mathrm{p}=0.19^{\star}$ \\
Prophylactic embolization & 1.47 & $0.37-5.96$ \\
Hemodynamic instability & 13.22 & $1.65-106.07$ \\
\hline
\end{tabular}

${ }^{*}$ p-values given where $95 \%$ CI not applicable

\section{DISCUSSION}

Endoscopy is the primary diagnostic and therapeutic modality for the management of upper and lower GI bleeding [10]. Common causes of non-variceal upper GI bleeding include ulcers, esophagitis, erosions, and Mallory-Weiss lesions, all of which are amenable to endoscopic treatment [11]. It is technically difficult for the endoscopist to access much of the small bowel and visualization of lesions can be limited in the presence of extensive hemorrhage or poor bowel preparation [12]. Failure of endoscopic management is positively associated with lesion number, size, location, and the amount of blood in the bowel $[9,13]$. It is estimated that up to $16 \%$ of upper GI and $25 \%$ of lower GI bleedings require invasive intervention beyond endoscopy using transcatheter arterial embolization (TAE) or surgery $[3,5]$. TAE is preferred to surgical interventions as it is less invasive and preserves the bowel [14-16].

The role of angiography is larger in lower GI bleeding than in upper GI bleeding, where endoscopic failure is relatively rare. Angiography demonstrates luminal extravasation or mucosal blush in up to $61 \%$ cases of suspected upper GI bleeding and in up to $50 \%$ of acute lower GI bleeds [6]. Initial technical success rates of TAE are as high as $93 \%$ for upper GI bleedings [9]. These numbers are similarly high for lower GI bleeding; 95\% initial success of embolization and 76\% remaining free of bleeding after 30 days [8]. Previous studies have shown negative angiograms with failure to localize active extravasation in as many as $52 \%$ of cases $[6,7]$. The low rate of positive angiographic studies is a more vexing problem in the management of lower GI bleeding than in upper GI bleeding, where empirical embolization has been found to be safe and effective. Selection of appropriate patients for angiography is a critical component of GI bleeding management due to the relatively low sensitivity of the procedure, the risk, albeit low, associated with angiography and the need for contrast exposure [17]. 
A number of clinical and laboratory parameters have been studied as predictors of extravasation and outcome in GI bleedings. Our study found that the drop in $\mathrm{Hb}$, rather that the absolute $\mathrm{Hb}$ level is an independent predictor of a positive angiogram in GI bleeding (OR 1.65, CI 1.128-2.403, $\mathrm{p}=0.010$ ). For every unit drop in the $\mathrm{Hb}$ value, there is a $65 \%$ increase in the odds of finding active extravasation on angiogram. Other investigators have found that the number of units of packed red blood cells and amount of fresh frozen plasma transfused in the 24 hours before angiography were positively associated with positive extravasation on angiography, with each unit of pRBC transfused increasing the probability of a positive angiographic study by $30 \%$ [18]. Abbas et al. [19] found that patients with lower GI bleeding requiring more than 5 units of blood in the 24 hours preceding angiography were significantly more likely to demonstrate extravasation on angiography. Hemodynamically unstable patients (systolic blood pressure at time of angiography less than $90 \mathrm{~mm}$ $\mathrm{Hg}$ ) have also been found by multiple investigators to be significantly more likely to demonstrate extravasation on angiography [7, 20, 21]. Interestingly, one group found that angiographies performed outside of standard working hours at their center's interventional center were more likely to demonstrate extravasation [22].

One of the important limitations of angiographic intervention in GI bleeding is the occurrence of rebleeding after embolization. In a review of 15 studies with 819 patients with upper GI bleeding, treated with angiographic intervention, the rate of rebleeding was documented at $33 \%$ : half of bleedings responded to repeat embolization [9]. Similarly, in a review of 15 studies with 309 patients with lower GI bleeding, the rate of rebleeding was estimated at $21 \%$ [8]. The rate of rebleeding in this study was $36 \%$, which is comparable to the above studies. Patients younger than 60 years were slightly less likely to have rebleeding compared to those over the age of 60 (OR $0.95, \mathrm{p}=0.04)$. Patients with known malignancy were at least 4 times more likely to rebleed within 30 days of procedure (OR $4.4, \mathrm{p}=0.04$ ), which is consistent with the findings of others demonstrating a strong proclivity for malignancy-associated GI bleeding to recur [23]. Interestingly, in one small study of patients with malignancy-associated GI bleeding, in which a majority of patients experienced recurrent bleeding after embolization, all recurrent bleedings occurred at sites other than the initial, embolized, source of bleeding [24].

The overall mortality from GI bleeding ranges from 5-12\% [4]. In a study of 362 patients with upper GI bleeding, Kelbl et al. [25] showed the mortality for hospital inpatients to be $39.0 \%$ compared to $11.1 \%$ for outpatients. Our study found a 30 -day mortality rate of $20.7 \%$ and the presence of hemodynamic instability prior to the angiogram was associated with a 13 times increased odds of death within 30-days. Rebleeding within 30 days was associated with more than 45 times increased odds of 30-day mortality (OR 46.8). Also, as expected, the presence of malignancy was an independent predictor of mortality. Interestingly, the number of units of platelets transfused was a predictor of mortality (OR 1.42). This is likely related to the comorbidities associated with thrombocytopenia rather than the effects of platelet transfusion itself. Comorbidities, especially malignancies are well known as a predictor of poor outcomes in acute GI bleeding [20,21].

In addition to clinical and laboratory assessments, many diagnostic imaging modalities have been studied for their usefulness in triaging GI bleeding patients for angiography. Diagnostic imaging modalities for GI bleeding include NM scintigraphy, computed tomographic angiography (CTA), and angiography. Historically, the 99-Tc tagged red blood cell scan has been the primary modality for localizing bleedings that are not seen by endoscopy. The sensitivity and specificity of this method have been reported as ranging from $78.6 \%$ $97 \%$ and $70.4 \%-100 \%$, respectively [26]. Though extremely sensitive, the length of time required for this study makes scintigraphy of limited utility in severe acute GI bleedings [27]. Furthermore, the anatomic quality of information obtained from the positive studies is limited, with $22 \%$ of positive studies being falsely localizing [28]. The utilization of tagged red cell scans was low in this cohort $(42,37 \%)$ and its sensitivity and specificity for identifying extravasation on angiography were $57.1 \%$ and $39.5 \%$, respectively. A tagged red blood cell study had a negative predictive value for a positive angiography of $83.3 \%$, and a positive predictive value of $14.8 \%$. This study showed that a positive tagged red cell scan is not a reliable predictor of active extravasation on angiography. This finding is related to the different sensitivity detected by two studies, where red cell scan can detect bleeding rate as low as $0.04 \mathrm{~mL} / \mathrm{min}$ compared to angiography which needs bleeding rate of $0.5 \mathrm{~mL} / \mathrm{min}$ to be positive $[29,30]$. Similar to these findings, prior studies have shown that tagged red blood cell scintigraphy is not helpful as a screening tool for selecting patients who are most likely to benefit from angiography [31].

CT angiography has more recently become more popular for the evaluation of GI bleeding as it is rapid, noninvasive, and allows accurate localization of bleeding site. A meta-analysis including 672 patients found CTA to have a sensitivity and specificity of $85 \%$ and $92 \%$, respectively, for the detection of active bleeding [32]. One small study found CTA to be somewhat helpful in triaging patients for angiography: out of their patients with negative CTA, $80 \%$ experienced spontaneous resolution of bleeding without further intervention, while $80 \%$ of patients with a positive CTA required endovascular or surgical intervention [33]. The rate of CTA utilization prior to angiography was very low in our cohort (17 patients, $15 \%$ ). Our study found CTA to have a sensitivity and specificity for positive angiography of $40 \%$ and $76.9 \%$, respectively. The positive predictive value of CTA for positive angiography was $40 \%$ and the negative predictive value was $76.9 \%$. The low numbers of CTA utilized in the series makes these numbers less reliable.

The main limitation of our study is its retrospective design. The timing between the presentation of the acute bleeding and angiography was not investigated and this could potentially lower the rate of detection of active extravasation in case of long delays or intermittent bleeding. Similarly, the timing between CTA, NM, and angiography was not measured and could have had an impact on sensitivity and specificity of CTA and NM on angiography. 


\section{CONCLUSION}

This study shows that the degree of drop in $\mathrm{Hb}$ from baseline, rather than absolute $\mathrm{Hb}$ value is an independent predictor of active extravasation on angiogram, with a 65\% increase in the odds of extravasation for every unit of $\mathrm{Hb}$ drop. Age less than 60 years was a negative predictor of rebleeding and malignancy increased the risk of rebleeding by more than four times. Rebleeding within 30 days of an angiography is a significant predictor of mortality, which increased the odds of death by 45 times. The presence of hemodynamic instability before angiogram increased the risk of 30-day mortality by 13 times. As might be expected, malignancy was also an independent predictor of 30-day mortality.

Conflicts of interest: None of the authors have any conflicts of interest to declare.

Authors' contributions: P.M., S.V. and G.N. contributed to study design and planning, analysis of imaging, and carried out the interventions concerned. J.M. compiled and analyzed data and wrote the greater part of the manuscript. A.B.D. contributed to data analysis and the writing and proofing of the manuscript. M.L. contributed to the statistical analysis of the data. A.N. and R.H. contributed to data gathering and wrote an initial version of the manuscript.

\section{REFERENCES}

1. Kim BS, Li BT, Engel A, et al. Diagnosis of gastrointestinal bleeding: A practical guide for clinicians. World J Gastrointest Pathophysiol 2014;5:467-478. doi:10.4291/wjgp.v5.i4.467

2. Peery AF, Dellon ES, Lund J, et al. Burden of gastrointestinal disease in the United States: 2012 update. Gastroenterology 2012;143:1179-1187. e3. doi:10.1053/j.gastro.2012.08.002

3. van Leerdam ME. Epidemiology of acute upper gastrointestinal bleeding. Best Pract Res Clin Gastroenterol 2008;22:209-224. doi:10.1016/j.bpg.2007.10.011

4. Chung P, Kim KE. Epidemiology of Acute Gastrointestinal Bleeding. In: Kim KE, ed. Acute Gastrointestinal Bleeding: Diagnosis and Treatment. Totowa, NJ: Humana Press; 2003:3-7.

5. Imdahl A. Genesis and pathophysiology of lower gastrointestinal bleeding Langenbecks Arch Surg 2001;386:1-7. doi:10.1007/s004230000194

6. Miller M Jr, Smith TP. Angiographic diagnosis and endovascular management of nonvariceal gastrointestinal hemorrhage. Gastroenterol Clin North Am 2005;34:735-752. doi:10.1016/j.gtc.2005.09.001

7. Kim JH, Shin JH, Yoon HK, et al. Angiographically negative acute arterial upper and lower gastrointestinal bleeding: incidence, predictive factors, and clinical outcomes. Korean J Radiol 2009;10:384-390. doi:10.3348/kjr.2009.10.4.384

8. Weldon DT, Burke SJ, Sun S, Mimura H, Golzarian J. Interventional management of lower gastrointestinal bleeding. Eur Radiol 2008;18:857 867. doi:10.1007/s00330-007-0844-2

9. Loffroy R, Rao P, Ota S, De Lin M, Kwak BK, Geschwind JF. Embolization of acute nonvariceal upper gastrointestinal hemorrhage resistant to endoscopic treatment: results and predictors of recurrent bleeding. Cardiovasc Intervent Radiol 2010;33:1088-1100. doi:10.1007/ s00270-010-9829-7
10. Cooper GS, Chak A, Way LE, Hammar PJ, Harper DL, Rosenthal GE. Early endoscopy in upper gastrointestinal hemorrhage: associations with recurrent bleeding, surgery, and length of hospital stay. Gastrointest Endosc 1999;49:145-152. doi:10.1016/S00165107(99)70478-5

11. Strate LL, Gralnek IM. ACG Clinical Guideline: Management of Patients With Acute Lower Gastrointestinal Bleeding. Am J Gastroenterol 2016;111:755. doi:10.1038/ajg.2016.155

12. Cherian MP, Mehta P, Kalyanpur TM, Hedgire SS, Narsinghpura KS. Arterial interventions in gastrointestinal bleeding. Semin Intervent Radiol 2009;26:184-196. doi:10.1055/s-0029-1225661

13. Loffroy R, Estivalet L, Cherblanc V, et al. Transcatheter embolization as the new reference standard for endoscopically unmanageable upper gastrointestinal bleeding. World J Gastrointest Surg 2012;4:223-227. doi:10.4240/wjgs.v4.i10.223

14. Ang D, Teo EK, Tan A, et al. A comparison of surgery versus transcatheter angiographic embolization in the treatment of nonvariceal upper gastrointestinal bleeding uncontrolled by endoscopy. Eur J Gastroenterol Hepatol 2012;24:929-938. doi:10.1097/ MEG.0b013e32835463bc

15. Ripoll C, Banares R, Beceiro I, et al. Comparison of transcatheter arterial embolization and surgery for treatment of bleeding peptic ulcer after endoscopic treatment failure. J Vasc Interv Radiol 2004;15:447-450. doi:10.1097/01.RVI.0000126813.89981.B6

16. Eriksson LG, Ljungdahl M, Sundbom M, Nyman R. Transcatheter arterial embolization versus surgery in the treatment of upper gastrointestinal bleeding after therapeutic endoscopy failure. J Vasc Interv Radiol 2008;19:1413-1418. doi:10.1016/j.jvir.2008.06.019

17. Cohn SM, Moller BA, Zieg PM, Milner KA, Angood PB. Angiography for preoperative evaluation in patients with lower gastrointestinal bleeding: Are the benefits worth the risks? Arch Surg 1998;133:50-55. doi:10.1001/archsurg.133.1.50

18. Lee L, Iqbal S, Najmeh S, Fata P, Razek T, Khwaja K. Mesenteric angiography for acute gastrointestinal bleed: predictors of active extravasation and outcomes. Can J Surg 2012;55:382-838. doi:10.1503/ cjs. 005611

19. Abbas SM, Bissett IP, Holden A, Woodfield JC, Parry BR, Duncan D. Clinical variables associated with positive angiographic localization of lower gastrointestinal bleeding. ANZ J Surg 2005;75:953-957. doi:10.1111/j.1445-2197.2005.03582.x

20. Barkun AN. Do predictors of mortality in upper gastrointestinal bleeding include a weekend time of admission? Clin Gastroenterol Hepatol 2009;7:257-258. doi:10.1016/j.cgh.2008.12.013

21. Zimmerman J, Siguencia J, Tsvang E, Beeri R, Arnon R. Predictors of mortality in patients admitted to hospital for acute upper gastrointestinal hemorrhage. Scand J Gastroenterol 1995;30:327-331. doi:10.3109/00365529509093285

22. Peynircioglu B, Erkus F, Cil B, Ciftci T, Durhan G, Balkanci F. Mesenteric angiography of patients with gastrointestinal tract hemorrhages: a single center study. Diagn Interv Radiol 2011;17:368-373. doi:10.4261/13053825.DIR.3963-10.1

23. Schatz RA, Rockey DC. Gastrointestinal Bleeding Due to Gastrointestinal Tract Malignancy: Natural History, Management, and Outcomes. Dig Dis Sci 2017;62:491-501. doi:10.1007/s10620-016-4368-y

24. Zheng L, Shin JH, Han K, et al. Transcatheter Arterial Embolization for Gastrointestinal Bleeding Secondary to Gastrointestinal Lymphoma. Cardiovasc Intervent Radiol 2016;39:1564-1572. doi:10.1007/s00270016-1422-2 
25. Klebl FH, Bregenzer N, Schofer L, et al. Comparison of inpatient and outpatient upper gastrointestinal haemorrhage. Int J Colorectal Dis 2005;20:368-375. doi:10.1007/s00384-004-0642-0

26. Chen PN, Brown RK. False positive GI bleed on Tc-99m RBC scintigraphy due to ileal varices. J Radiol Case Rep 2012;6:23-28. doi:10.3941/jrcr.v6i2.844

27. Zurkiya O, Walker TG. Angiographic Evaluation and Management of Nonvariceal Gastrointestinal Hemorrhage. AJR Am J Roentgenol 2015;205:753-763. doi:10.2214/AJR.15.14803

28. Fallah MA, Prakash C, Edmundowicz S. Acute gastrointestinal bleeding. Med Clin North Am 2000;84:1183-1208. doi:10.1016/S00257125(05)70282-0

29. Zuckier LS. Acute gastrointestinal bleeding. Semin Nucl Med 2003;33:297-311. doi:10.1016/S0001-2998(03)00033-3
30. Baum S. Arteriographic Diagnosis and Treatment of Gastrointestinal Bleeding. In: Geschwind J, Dake M, eds. Abrams' Angiography. 2nd edn: Wolters Kluwer; 2006:488.

31. Pennoyer WP, Vignati PV, Cohen JL. Mesenteric angiography for lower gastrointestinal hemorrhage are there predictors for a positive study? Dis Colon Rectum 1997;40:1014-1018. doi:10.1007/BF02050921

32. Garcia-Blazquez V, Vicente-Bartulos A, Olavarria-Delgado A, et al. Accuracy of CT angiography in the diagnosis of acute gastrointestinal bleeding: systematic review and meta-analysis. Eur Radiol 2013;23:11811190. doi:10.1007/s00330-012-2721-x

33. Foley PT, Ganeshan A, Anthony S, Uberoi R. Multi-detector CT angiography for lower gastrointestinal bleeding: Can it select patients for endovascular intervention? J Med Imaging Radiat Oncol 2010;54:916. doi:10.1111/j.1754-9485.2010.02131.x 